\title{
EchoGéo
}

29 | 2014

The Political Ecology of Conservation and

Development Territories

\section{Enjeux d'une cartographie du patrimoine côtier du fond du bassin d'Arcachon (Gironde, France)}

Frédéric Bertrand, Lydie Goeldner-Gianella and Geneviève Decroix

\section{(2) OpenEdition}

Journals

Electronic version

URL: https://journals.openedition.org/echogeo/13948

DOI: $10.4000 /$ echogeo. 13948

ISSN: 1963-1197

Publisher

Pôle de recherche pour l'organisation et la diffusion de l'information géographique (CNRS UMR 8586)

Electronic reference

Frédéric Bertrand, Lydie Goeldner-Gianella and Geneviève Decroix, "Enjeux d'une cartographie du patrimoine côtier du fond du bassin d'Arcachon (Gironde, France)", EchoGéo [Online], 29 | 2014, Online since 27 October 2014, connection on 01 August 2021. URL: http://journals.openedition.org/echogeo/ 13948 ; DOI: https://doi.org/10.4000/echogeo.13948

This text was automatically generated on 1 August 2021.

EchoGéo est mis à disposition selon les termes de la licence Creative Commons Attribution - Pas d'Utilisation Commerciale - Pas de Modification 4.0 International (CC BY-NC-ND) 


\title{
Enjeux d'une cartographie du patrimoine côtier du fond du bassin d'Arcachon (Gironde, France)
}

\author{
Frédéric Bertrand, Lydie Goeldner-Gianella and Geneviève Decroix
}

Les auteurs remercient le programme Liteau du MEDDE, le Conseil général de Gironde et le Conseil régional d'Aquitaine pour le financement du programme "Barcasub", de même que le Conservatoire du littoral, propriétaire de certains domaines endigués mentionnés.

\section{Introduction}

1 La carte occupe une place de choix dans les représentations sociales du bassin d'Arcachon et la production des savoirs relatifs à cette vaste lagune du littoral ouesteuropéen $\left(174 \mathrm{~km}^{2}\right)$, située au creux du golfe de Gascogne. La simplicité des contours triangulaires du bassin, très tôt soulignée par les géographes (Waleursee, 1928), et l'instabilité des passes mettant en communication l'intérieur de la lagune et l'océan Atlantique, se prêtent à la figuration des traits distinctifs de cette vaste et unique échancrure du littoral landais. Mais la complexité du milieu arcachonais, liée à l'imbrication particulière des enjeux économiques et environnementaux dans un contexte de changement climatique et d'accroissement des risques côtiers d'érosion et de submersion marine, appelle tout autant le recours à la carte pour tenter de démêler l'écheveau des facteurs contribuant à la mise en valeur des biens naturels et culturels du bassin aux différents étapes du processus de patrimonialisation (prise de conscience, jeu d'acteurs, sélection d'éléments et justification des choix, actions de conservation et de restauration). En effet, cette patrimonialisation a pris, dans les dernières décennies, une orientation autant naturelle que culturelle, portant à la fois sur les rives du bassin (relative proximité de la dune du Pyla, forêt landaise...), le trait de côte (polders des $\mathrm{XVIII}^{\mathrm{e}}$ et $\mathrm{XIX}^{\mathrm{e}}$ siècles $^{1}$, schorres...) et sa partie centrale (Ile aux Oiseaux, cabanes tchanquées, habitats benthiques...), trois secteurs attirant une avifaune importante. Cartographier ce patrimoine permet non seulement de mettre en évidence son 
maintien, ainsi que son état, mais aussi de réfléchir à d'éventuelles concurrences entre éléments patrimoniaux du même type (naturels ou culturels) ou distincts (naturels et culturels). Après un bref aperçu de l'état de l'art cartographique dédié au bassin d'Arcachon, nous détaillons les objectifs et les fondements méthodologiques de la carte proposée, avant de montrer en quoi elle constitue un outil potentiel de réflexion et de communication sur les enjeux de la patrimonialisation dans le bassin d'Arcachon.

\section{Bref éclairage sur l'état de l'art cartographique du bassin d'Arcachon}

2 En dépit de la forme triangulaire simple et ramassée du bassin d'Arcachon, il a fallu attendre le début du XVIII ${ }^{e}$ siècle et la publication par l'ingénieur Claude Masse d'une première carte pour disposer d'une vue d'ensemble de la structure générale et de l'organisation interne du bassin (Bousquet-Bressolier et al., 1990). Dès lors, la représentation de la "dualité " géographique du bassin d'Arcachon, remarquable autant par la fixité de ses contours dans le contexte hydrodynamique régional ${ }^{2}$ que par la mobilité de ses paysages largement anthropisés, n'a cessé de s'affiner en fonction de l'évolution des préoccupations scientifiques et des techniques de production cartographique. La représentation cartographique du terrain arcachonnais va évoluer au cours de trois phases marquantes en termes d'innovations techniques et de développement des sciences naturalistes ainsi qu'en fonction de l'incorporation progressive des considérations environnementales dans la valeur accordée au milieu lagunaire (enjeux) et dans l'évaluation de son évolution (indicateurs de suivi).

3 La première phase, entre la publication de la carte de Masse (1708) et celle de l'hydrographe Beautemps-Beaupré (1826), en passant par la publication de carte de Clavaux (1774 et 1776), a permis, grâce à la qualité de leurs levés et de leur exécution, de donner la mesure des transformations paysagères liées aux endiguements et, ainsi, du caractère terrien de cet espace littoral désormais en partie poldérisé (BousquetBressolier et al., 1990 ; Goeldner-Gianella et al., 2003).

4 Une seconde étape dans l'évolution de la représentation du bassin d'Arcachon est liée au développement des recherches en biologie marine à partir de la fondation de la société Biologique d'Arcachon (1863) et, surtout, de la création de la station biologique d'Arcachon (1867) appelée à devenir un centre d'océanographie biologique d'envergure nationale. La publication de la Carte marine des chenaux, bancs de sable et crassats ${ }^{3} d u$ bassin au 1/20.000 dans le cadre d'une thèse en océanographie présentée à la faculté des sciences de l'Université de Bordeaux (Bouchet, 1968) offre ainsi la première vision détaillée et complète de l'hydrographie du bassin intégrant pleinement les aménagements des rives. Une série de travaux améliorant de façon significative la connaissance du milieu lagunaire (Amanieu, 1967; Bouchet et al., 1983 ; Carruesco \& Alvinerie, 1990 ; Auby, 1991) permettront de compléter cette carte physiographique par la Carte de l'environnement marin dans le bassin d'Arcachon au 1/25.000 (Bouchet J.-M., 1998). En dépit de l'ancienneté des couvertures photographiques utilisées pour délimiter les unités bio-sédimentaires qualifiées à partir des peuplements naturels caractéristiques, cette carte en couleurs ${ }^{4}$ offre de nos jours la vision la plus complète du milieu du bassin d'Arcachon. La reconnaissance de ce document par les scientifiques aussi bien que par les gestionnaires est d'autant plus remarquable que l'adoption de représentations conventionnelles n'y exclut pas la volonté de se conformer à une 
imitation de la nature (utilisation de points pour le sable grossier constitutif des barres de méandre, de signes pour la végétation "moutonnante" des schorres $\left.{ }^{5} . ..\right)$ au risque de restituer localement une illusion de relief comme sur les cartes anciennes.

Enfin, la représentation et la connaissance du bassin d'Arcachon ont été profondément renouvelées depuis les années 1990, comme celles de l'ensemble des littoraux du globe, par la diffusion et l'utilisation des données satellitales. Dans le contexte d'instabilité morphodynamique et de forte pression anthropique, les applications de la télédétection ont porté en priorité sur la surveillance des passes à travers l'analyse diachronique de l'évolution morphologique sous-marine (Froidefond, 2006) et le suivi des habitats benthiques côtiers ${ }^{6}$, en particulier des herbiers de Zostères en forte régression dans certaines parties de la lagune (Blanchet, 2004 ; Lafon et al., 2002 ; Lafon et al., 2008 ; Auby et al., 2011). L'effort d'inventaire cartographique a été étendu aux habitats côtiers en lien avec l'élaboration des documents d'objectifs Natura2000 ou la restauration écologique des secteurs dépoldérisés de Graveyron et de Malprat situés en fond de bassin (Bertrand \& Goeldner, 2013). Le couplage de données spatiales à haute résolution et de données de terrain acquises selon un plan d'échantillonnage serré a permis d'affiner la connaissance des processus évolutifs et favorisé une multiplication de cartes d'analyse (géomorphologie, biogéographie) et de cartes de systèmes (cartes de risques naturels) sans toutefois modifier fondamentalement la vision d'ensemble de la lagune.

6 Ainsi, la nécessité d'élaborer une "carte du patrimoine côtier du fond du bassin d'Arcachon" est apparue évidente dans le contexte cartographique d'un littoral historiquement renseigné mais inégalement documenté sur les dynamiques spatiales actuelles liées à la gestion du risque de submersion et à l'émergence d'enjeux patrimoniaux dans le fond du bassin.

\section{Objectifs et fondements méthodologiques d'une cartographie du patrimoine côtier}

7 Par rapport aux cartes susmentionnées, réalisées selon une approche analytique visant à cartographier des éléments (formations superficielles) ou des processus (dégradation des herbiers) simples, la carte du patrimoine côtier du fond du bassin d'Arcachon vise à une représentation synthétique des problèmes liés à la répartition des hommes et des enjeux présents dans une portion de territoire donnée, plus particulièrement dans le cas des enjeux patrimoniaux. Conçue comme une déclinaison de la carte de l'Environnement telle qu'elle a été définie dans les années 1980 par les géographes (Journaux, 1985), elle est destinée à faire prendre conscience des problèmes liés à la mise en patrimoine des éléments naturels et culturels de la partie interne du bassin d'Arcachon en rapport avec l'inscription progressive du secteur sous divers inventaires ou statuts de protection (Goeldner-Gianella \& Bertrand, 2014). Elle doit, ce faisant, aider les responsables et gestionnaires de l'environnement à agir à bon escient en fonction d'objectifs de gestion patrimoniale tels que ceux prévalant dans la gestion des polders protégés de Certes, de Graveyon et de l'île de Malprat.

8 Les finalités de cette carte de synthèse ont orienté sa conception, fondée non pas sur l'intégration des facteurs physiques et humains en un nombre plus ou moins élevé de combinaisons mais sur la juxtaposition et la superposition de l'ensemble de ces 
facteurs. Les principales difficultés de sa réalisation résident, d'une part, dans l'abandon des facteurs qui, sans être négligeables dans le questionnement représenté sur la carte, risqueraient par leur trop grand nombre de rendre celle-ci illisible et, d'autre part, dans la nécessité de conférer à cette carte un caractère dynamique qui lui permette de traduire et de visualiser des phénomènes de patrimonialisation dans la durée aussi bien que dans l'espace. Il a ainsi été décidé de ne pas intégrer à la carte d'informations sur l'état des schorres de bordure, la nature et la hauteur des digues, les niveaux de marée, l'orientation des courants et du fetch, le statut foncier des polders.

Le choix de l'aire à cartographier s'est porté sur la zone d'étude du projet de recherches BARCASUB - projet financé par le programme de recherche Liteau consacré à l'analyse et la gestion du risque de submersion marine dans le bassin d'Arcachon dans la mesure où le processus de patrimonialisation s'y trouve largement associé à l'émergence de nouveaux enjeux autour de la question de la dépoldérisation. L'échelle de représentation initiale au $1 / 25.000$ a été choisie en fonction de la dimension spatiale des biens naturels et culturels à valeur patrimoniale identifiés sur le terrain et correspond à celle de la Carte de l'environnement marin dans le bassin d'Arcachon mentionnée ci-dessus.

Les paramètres retenus dans la cartographie du patrimoine sont :

- les données relativement stables du milieu physique conditionnant la distribution actuelle des habitats benthiques côtiers à valeur patrimoniale, comme la morphologie des vasières intertidales (alias slikke ou crassat) et des chenaux de marée associés,

- les biens naturels et culturels à valeur patrimoniale reconnue tels les prairies humides ou des bâtiments (cabanes d'éclusier, grange, habitation) dispersés autour des différents domaines et figurés en rouge,

- les facteurs d'évolution de la valeur patrimoniale des sites côtiers liés à la submersibilité des rives, en rapport notamment avec le niveau de submersion attendu en 2100 , et à la fréquentation des digues défensives par des promeneurs dans leur grande majorité et quelques véhicules légers - deux paramètres analysés et cartographiés en détail dans le cadre du projet BARCASUB (Bertrand \& Goeldner, 2013). 


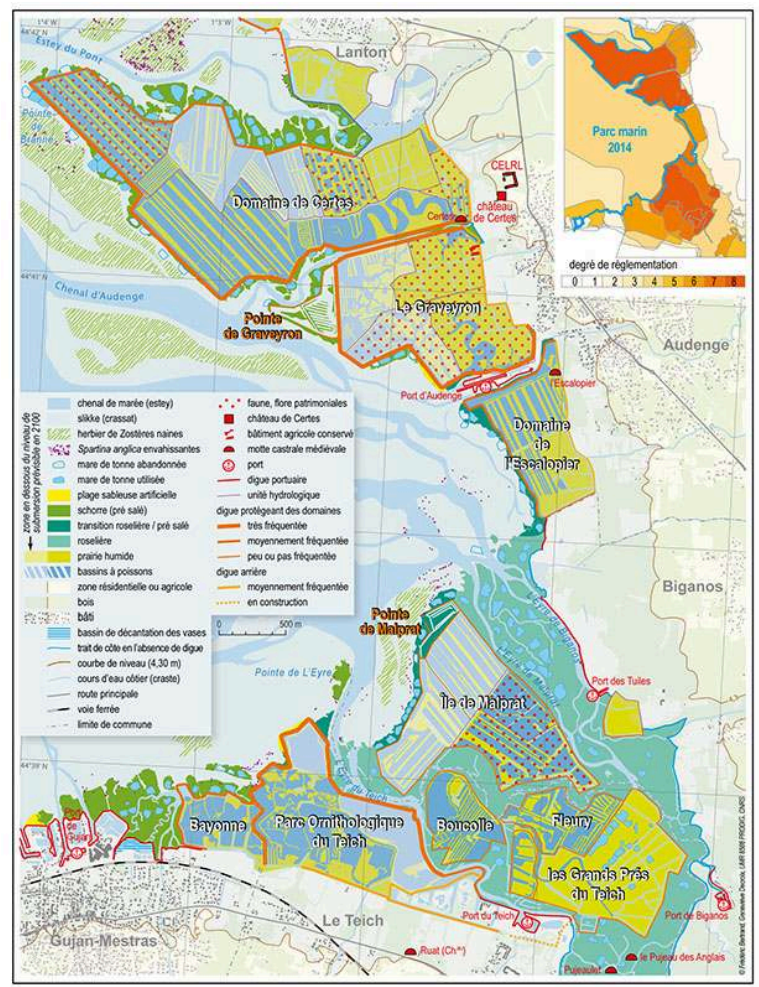

Pour obtenir la carte au format PDF (1 Mo), se reporter à la rubrique documents annexes. Auteurs : Fréderic Bertrand, Geneviève Decroix.

\section{Commentaire de la carte et apport à la compréhension du phénomène de patrimonialisation}

La carte du patrimoine attire d'abord l'attention par l'extension des éléments résiduels de végétation naturelle de marais représentée par des aplats de vert à proximité d'agglomérations urbaines en cours de coalescence (Lanton, Audenge, Biganos, Le Teich, Gujan-Mestras). Dans ce contexte d'urbanisation rapide (taux de croissance des communes compris entre 2 et $3 \%$ par an dans les dernières décennies), le delta de la Leyre et, à un degré moindre, le plateau forestier qui accompagne les ruisseaux de Lanton, du Milieu et de Passaduy, apparaissent comme des coupures d'urbanisation entre le bassin et la forêt landaise, dans lesquelles se concentrent les enjeux patrimoniaux du fond du bassin. Le Parc Ornithologique du Teich (ROT $\left.{ }^{7}\right)$ s'inscrit sur la marge de cet espace deltaïque intra-lagunaire. Sa création en 1972 à l'emplacement d'un ancien polder a été déterminante dans l'essor d'un tourisme de nature très différent de celui d'Arcachon et dans la promotion du fond du bassin en "cœur de bassin", selon l'expression utilisée par les acteurs touristiques, en vertu d'un patrimoine naturel d'exception. Celui-ci est aujourd'hui valorisé par l'ouverture au public de nombreux sites dont les digues des polders de Certes, Graveyron, Malprat ou Bayonne sur un linéaire d'une trentaine de kilomètres. L'engouement pour la richesse naturelle $\mathrm{du}$ fond du bassin est lié également à son inscription progressive depuis deux décennies sous divers inventaires ou statuts de protection (sites classés et inscrits, ZNIEFF I, PNR, espaces remarquables de la Loi Littoral, Natura2000, Site Ramsar, PNM) 
très largement imbriqués et dont le carton complémentaire met en évidence qu'ils confèrent aux secteurs poldérisés le plus haut degré de protection réglementaire. Les habitats benthiques et côtiers du domaine lagunaire stricto sensu, situé en deçà des digues de mer, donc à l'extérieur du secteur estuarien de la Leyre dominé par les roselières, bénéficient également, depuis peu (juin 2014), de la protection du parc naturel marin du bassin d'Arcachon.

La double question de la valorisation du patrimoine et du maintien de l'intégrité patrimoniale de ces territoires côtiers se pose à l'aune de plusieurs variables représentées sur la carte dont, au premier chef, la fréquentation des digues protégeant les domaines. Bien que très inégale, comme le montrent des comptages effectués dans le cadre du programme BARCASUB transcrits par des variations dans l'épaisseur du trait, cette fréquentation atteste $d u$ fort attachement des populations locales et de l'intérêt des touristes pour ces domaines - tous deux liés au caractère très attractif des paysages offerts à la vue des promeneurs des deux côtés externe (maritime) et interne (terrestre) des digues (Goeldner-Gianella et al., 2013). La richesse floristique et/ou faunistique de certains casiers endigués, renseignée ici à dire d'experts et soulignée sur le terrain par les actions de valorisation publique (panneaux, visites) du Conservatoire du Littoral ou des gestionnaires, renforce l'intérêt pour le patrimoine naturel des sites visités. Pour autant, la valeur patrimoniale de ces sites "en bout de terre" bien que situés en fond de bassin ne procède pas seulement de leur "pittoresque écologique" (Luginbühl, 20018). Dans un contexte d'artificialisation très poussée du paysage dont rend compte la trame serrée des aménagements hydrauliques, les enjeux patrimoniaux, outre les habitats et paysages naturels, procèdent aussi du bâti (bâtiments agricoles, cabanes d'éclusier), des ouvrages de défenses (digues, mottes castrales) et des éléments constitutifs des casiers (bosses, plats et fossés). Ainsi, les secteurs Ouest et surtout Nord-Ouest du domaine de Certes, moyennement fréquentés en dépit d'un patrimoine naturel reconnu, n'en présentent pas moins une valeur patrimoniale élevée liée à l'entretien de longs plats 9 orientés initialement face au vent dominant pour favoriser l'évaporation des surfaces en eau et évoquant l'ancien usage salicole de ce polder.

13 Toutefois, le patrimoine côtier est en cours de requalification compte tenu de l'exposition particulière $d u$ fond du bassin aux effets submersifs du relèvement du niveau marin et de la réponse adaptative des populations au risque croissant de submersion au cours des prochaines décennies. La cote 4,30 m NGF représente le niveau d'eau extrême à la côte qui serait fonction du niveau de surcote atteint lors de l'aléa de référence Xynthia $(3,70 \mathrm{~m})$ doublé d'une élévation du niveau de la mer de $60 \mathrm{~cm}$ à

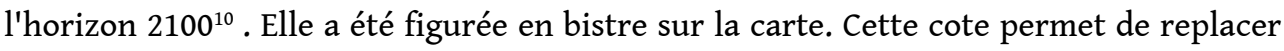
la dynamique de patrimonialisation dans le contexte de la politique nationale de gestion des zones basses urbanisées (Goeldner-Gianella \& Bertrand, 2014). Si l'on suit le principe «d'inconstructiblité à géométrie variable» - qui prend en considération l'élévation prévisible du niveau de la mer aussi bien que l'occupation effective du sol édicté par les textes réglementaires post-Xynthia ${ }^{11}$, le fond du bassin ressort clairement comme une zone où l'urbanisation restera limitée par le seul fait d'être considérée, au vu de la réglementation, comme inconstructible.

14 La mise en valeur et la conservation effectives de ce patrimoine n'en demeurent pas moins subordonnées à d'autres paramètres tels que le contrôle de l'urbanisation et 
l'élévation du niveau marin. Certains de ces paramètres sont simplement suggérés par la carte, tels :

- l'exposition différentielle des rives à la propagation du clapot suggérée par l'ouverture dans les digues les plus exposées des pointes de Graveyron et de Malprat (Bertrand, 201 ; Bertrand et al., 2014),

- la dynamique régressive des herbiers de Zostères naines (Auby et al., 2011) suggérée par le recouvrement discontinu des slikkes, l'envahissement des estrans par les Spartinaies invasives à Spartina anglica (Le Nindre et al., 2006) indiquée par le paysage punctiforme au front du delta de la Leyre et dans la zone externe de l'estey du Pont ou encore

- le mitage des schorres de front de digue par les mares de chasse.

15 Ces facteurs dynamiques interagissent dans le fonctionnement et l'évolution d'un système bio-sédimentaire fortement anthropisé dans lequel l'imbrication des milieux terrestres et marins et la gamme des habitats naturels et semi-naturels correspondants dépend aussi étroitement des actions de gestion patrimoniale entreprises par les différents acteurs locaux (ROT, Conservatoire du Littoral, Conseil général de Gironde, associations, chasseurs).

\section{Conclusion}

La représentation combinée d'informations patrimoniales liées aux aspects paysagers et à la fréquentation des rives du fond du bassin d'Arcachon sur une même carte à une date donnée aide ainsi à effectuer des choix de gestion du littoral privilégiant un élément patrimonial aux dépens d'un autre. Deux secteurs spécifiques de ce fond de bassin résument à eux seuls les enjeux d'une cartographie de l'état actuel du patrimoine côtier et de son devenir : ce sont les zones accidentellement rendues à la mer nommées sur la carte Pointe de Graveyron et Pointe de Malprat. Faut-il accepter ou non la rupture accidentelle de digue qui s'est produite lors de tempêtes passées - et donc le retour de la mer dans des zones historiquement endiguées et exploitées ? Cette question renvoie à celle, cruciale de la nature du patrimoine à préserver : faut-il préférer la renaissance d'un patrimoine naturel de slikke et de schorre à la conservation d'un patrimoine culturel ici constitué de bassins à poissons - mais qui comprend, en d'autres secteurs des polders, des prairies humides et des bâtiments agricoles? Si l'option du retour de la mer et de la reconquête végétale de la zone dépoldérisée est retenue, est-on certain de ne pas simultanément favoriser l'extension de la spartine anglaise, plante certes conquérante sur un bas-schorre, mais également invasive - et non éloignée de la Pointe de Malprat? Ce patrimoine naturel sera-t-il à même de résister aux effets des submersions à venir et de remplacer en cela les anciennes digues, jusqu'à se transformer en infrastructure naturelle de défense contre la mer, permettant le maintien hors d'eau des surfaces endiguées voisines? La présence d'un patrimoine culturel, même en ruine (ici les anciennes digues), ne peut-elle contribuer à accroître cette résistance potentielle aux forces marines? Ces questions sont mises en exergue par la superposition, dans une base de données spatialisée, d'informations rarement représentées simultanément dans des documents de gestion et à une échelle facilitant la communication. Elles pourront ainsi être discutées plus avant avec les acteurs locaux de la gestion environnementale. La carte aidera aussi à élargir cette réflexion à l'ensemble des zones dépoldérisables du fond du bassin d'Arcachon. 


\section{BIBLIOGRAPHY}

Amanieu M., 1967. Recherches écologiques sur les faunes des plages abritées et des étangs saumâtres de la région d'Arcachon. Thèse de doctorat, Faculté des Sciences Bordeaux, nº 197, 2 vol.

Auby I., 1991. Contribution à l'étude des herbiers de Zostera noltii dans le Bassin d'Arcachon: Dynamique, production et dégradation, macrofaune associée. Thèse de l'Université de Bordeaux I, 234 p.

Auby I., Bost C.-A., Budzinski H., Dalloyau S., Desternes A., Belles A., Trut G., Plus M., Pere C., Couzi L., Feigné C., Steinmetz J., 2011. Régression des herbiers de zostères dans le Bassin d'Arcachon : état des lieux et recherche des causes. Rapport de contrat, 156 p. + annexes. http:// archimer.ifremer.fr/doc/00054/16507/

Bertrand F., Goeldner-Gianella L., Anselme B., Durand P., Thomas Y.-F., Sottolichio A., Sénéchal N., 2014. L'aléa submersion marine sur la rive interne du Bassin d'Arcachon : exposition actuelle, cartographie prévisionnelle et capacité d'atténuation par la restauration des marais salés. Actes Colloque international : Connaissance et Compréhension des Risques Côtiers : Aléas, Enjeux, Représentations, Gestion, p. 124-134. http://cocorisco.sciencesconf.org/

Bertrand F., 2013. The Arcachon Bay estuary: a "collage" of landscapes. In M. Fort and M.-F. André (eds.), Landscapes and Landforms of France, World Geomorphological Landscapes, p. 71-80. http://link.springer.com/chapter/10.1007/978-94-007-7022-5_8

Bertrand F., Goeldner-Gianella L., 2013. BARCASUB : La SUBmersion marine et ses impacts environnementaux et sociaux dans le Bassin d'ARCAchon (France) : est-il possible, acceptable et avantageux de gérer ce risque par la dépoldérisation? Rapport final. Ministère de l'Ecologie, du Développement durable et de l'Energie (MEDDE), 178 p . + annexes. Présentation au colloque de Boulogne-sur-Mer (France), 21-22 nov. 2013. http://www1.liteau.net/uploads/article/Projet-BARCASUB.pdf

Blanchet H., 2004. Structure et fonctionnement des peuplements benthiques du Bassin d'Arcachon. Thèse de doctorat, Université Bordeaux I, $331 \mathrm{p}$.

Bouchet J.-M., 1968, Etude océanographique des chenaux du bassin d'Arcachon, Thèse de doctorat, Faculté des Sciences Bordeaux, $\mathrm{n}^{\circ}$ 212, 2 vol., 370 p.

Bouchet J.-M., 1998. Carte de l'environnement marin dans le bassin d'Arcachon. IFREMER, Bordeaux.

Bouchet J.-M., Bachelet G., Cornet M., Lissalde J. P., Sorbe J. C., Amoureux L., 1983. Évolution des peuplements des fonds côtiers au large du bassin d'Arcachon $i$ Littoral soft bottom communities evolution off the Arcachon Bay. Oceanologica Acta, 17e symposium européen de biologie marine, Brest, 27 septembre 1982, pp. 39-4

Bousquet-Bressolier C., Bouscau F., Pajot M.-J., 1990. Les aménagements du bassin d'Arcachon au XVIIIème siècle. Mémoire EPHE n 43, 224 p.

Carruesco C., Alvinerie J., 1990. Le bassin d'Arcachon, environnement lagunaire de la côte landaise en constante évolution. Actes colloque Arcachon, octobre 1990, Soc. Histor. et Archéol. d'Arcachon et du Pays de Buch "Le littoral gascon et son arrière pays".

Froidefond, J.-M., 2006. Evolution des passes du bassin d'Arcachon. Conférence le 11 octobre 2006 à Gujan-Mestras.

Goeldner-Gianella L., Anselme B., Bertrand F., Durand P., 2003. Impacts du changement climatique sur l'intervention du Conservatoire du Littoral, recul du trait de côte et orientations stratégiques, Domaine de Graveyron (bassin d'Arcachon, Gironde. 48 p. 
Goeldner-Gianella L., Bertrand F., Pratlong F., Gautier-Gaillard S., 2013. Submersion marine et dépoldérisation : le poids des représentations et des pratiques sociales dans la gestion du risque littoral. Espace, Populations, Sociétés, ${ }^{\circ}$ 1/2, p. 193-209.

Goeldner-Gianella L. \& Bertrand F., 2014 (à paraître). Gérer le risque de submersion marine par la dépoldérisation : représentations locales et application des politiques publiques dans le bassin d'Arcachon. Natures Sciences Sociétés.

Journaux A., 1985. Cartographie intégrée de l'environnement : un outil pour la recherche et pour l'aménagement. Coll. Notes techniques du MAB, Paris, UNESCO, 56 p.

Lafon V., Froidefond J.-M., Lahet F., Castaing P., 2002. SPOT shallow water bathymetry of a moderately turbid tidal inlet based on field measurements. Remote Sens. Environ., 8, p. 136-148.

Lafon V., Marieu V., Butel R., Dehouck A., Froidefond J.-M.,Trut G., 2008. Cartographie des faciès bio-sédimentaires du Bassin d'Arcachon à partir de l'imagerie FORMOSAT-2. Xes Journées Nationales Génie Côtier - Génie Civil, 14-16 octobre 2008, Sophia Antipolis, p. 563-572.

Waleursee J., 1928. Le bassin d'Arcachon. Annales de Géographie. t. 37, n² 209. p. 407-427.

\section{NOTES}

1. Ces polders sont appelés localement les "Domaines endigués".

2. A l'exception notable de la Leyre dont le bassin d'Arcachon constitue le débouché estuarien, tous les cours d'eau de la côte landaise ont évolué naturellement en étangs littoraux.

3. Terme vernaculaire du bassin d'Arcachon, désignant comme le terme de slikke, la partie inférieure des étendues intertidales et se présentant comme une vasière molle et dépourvue de végétation, constituée de sédiments fins fortement imprégnés d'eau (cf. légende de la carte ici présentée).

4. Depuis peu numérisée et consultable en ligne sur l'infrastructure de données géographiques marines et littorales de l'IFREMER (http://sextant.ifremer.fr/fr/ )

5. Partie supérieure des étendues intertidales formées de sédiments fins accumulés par les pleines mers et couverte d'une végétation halophile dense et basse.

6. Catégorie d'habitats situés dans la zone de balancement des marées et les petits fonds côtiers accueillant des organismes : (algues, phanérogames, vers, mollusques, crustacés, poissons, etc..) vivant en relation étroite avec les fonds subaquatiques.

7. Désormais nommé "Réserve ornithologique du Teich".

8. http://www.ladocumentationfrancaise.fr/var/storage/rapports-publics/014000726/0000.pdf

9. Etendues plates et peu profondes des anciens marais à poissons s'opposant aux profonds.

10. Sur la base de l'hypothèse pessimiste de l'Observatoire National sur les Effets du Réchauffement Climatique.

11. Circulaires du Ministère de l'Écologie, de Énergie, du Développement Durable et de la Mer (MEEDDM) du 7 avril 2010 relative aux mesures à prendre suite à la tempête Xynthia et du 27 juillet 2011 qui introduit en plus de l'aléa de référence, un aléa à l'horizon 2100 intégrant une surélévation de $60 \mathrm{~cm}$ sur la base de l'hypothèse « pessimiste » de l' Observatoire National sur les Effets du Réchauffement Climatique (ONERC). 


\section{AUTHORS}

\section{FRÉDÉRIC BERTRAND}

Frédéric Bertrand, Frederic.Bertrand@paris-sorbonne.fr, est Professeur de géographie à

l'Université Paris-Sorbonne (Paris 4) et membre de l'UMR 8586 PRODIG.

\section{LYDIE GOELDNER-GIANELLA}

Lydie Goeldner-Gianella, Lydie.Goeldner-Gianella@univ-paris1.fr, est Professeure de Géographie à l'Université Paris 1 - Panthéon-Sorbonne, UMR 8586 PRODIG.

\section{GENEVIÈVE DECROIX}

Geneviève Decroix est Ingénieur d'études, à l'UMR 8586 PRODIG. 